\title{
LAS PULSIONES Y LAS DROGAS
}

Drives and Drugs

Julián Andrés Hernández Gómez jahernandezgom@poligran.edu.co

Leidy Angélica Hernández lehernandez16@poligran.edu.co

Sergio Nicolás Riveros Correo electrónico

Institución Universitaria Politécnico Grancolombiano

Psicología

Bogotá D.C, Colombia

\section{Resumen}

El presente estudio busca identificar y describir las pulsiones de personas entre los 18 y 35 años relacionadas con el consumo de sustancias psicoactivas en la ciudad de Bogotá. Las personas serán escogidas usando un muestreo por intensidad, y serán elegidas del ejercicio etnográfico de observación no participante en espacios recreativos y de fiesta que presentan el fenómeno. Además, se realizarán entrevistas con guion de la entrevista y un posterior análisis inductivo-deductivo. Al ser un estudio que se encuentra en desarrollo tanto la discusión como las conclusiones son parciales y se basan en el abordaje de los datos recolectados hasta ahora llegando a la conclusión que las sustancias como objeto logran generar siempre el esfuerzo suficiente para llegar a la meta, aunque dependiendo de la fuente pueden afectar el desarrollo de los componentes de la pulsión.

Palabras clave: Pulsiones, Drogas, Estímulo pulsional, Energía Psíquica. 


\section{INTRODUCCIÓN}

Según la Oficina de las Naciones Unidas Contra la Droga y el Delito (2018) alrededor de 275 millones de personas en el mundo han consumido alguna sustancia psicoactiva ilegal, y de este número 13.8 millones de jóvenes entre 15 y 16 años consumieron cannabis, siendo esta es la droga que más se consume, y también entre muchas poblaciones es la primera droga ilegal que se prueba. Desde otra perspectiva, el Observatorio de Drogas de Colombia (2017), reporta que el consumo de sustancias ilícitas ha venido incrementando. Se estima que alrededor de tres millones de personas han consumido alguna vez en el país y, en la encuesta del 2013 es destacable el aumento en la prevalencia de uso alguna vez en la vida; pasando de $8,8 \%$ a $12,2 \%$, y el uso durante el último año pasando de 2,6\% en 2008 y 3,6\% en 2013.

Este tipo de estudios en personas consumidoras de sustancias no son tan comunes, en Colombia no existen referencias que analicen el consumo de sustancias desde las teorías Freudianas, y en general la mayor parte de literatura encontrada en este tema se centra en explicar el consumo problemático. Por estas razones propuestas como esta son un adelante en la comprensión del comportamiento del consumo que no necesariamente está relacionado con la enfermedad mental o la afectación en salud (Garrido, 2016; Guzmán, 2015; Obando, 2015; Peña \& Herrera, 2016).

La pulsión es definida como una fuerza, un empuje, que opera desde el interior de la persona, proviniendo de estímulos interiores(Daza-Orozco, 2019; Flórez-Romero Diana Paola; Castillo-Triana, Nicolás; Contreras-Ruiz, Paula Katerine; Henao-Floréz, Andrés; Ramírez-Toro, Diana Milena; Zárate-Pinto, Germán Camilo; GuzmánRodríguez, Rosa Julia; Anzelín-Zuluaga, Ingrid Carolina; Ruiz-He, Nicolás, 2020). Esta pulsión emerge como fuerza constante que no puede ser reprimida y termina generando la satisfacción de los estímulos pulsionales a través de actividades complejas y encadenadas que modifican el mundo exterior, lo suficiente para satisfacer dicha fuente interior del estímulo pulsional, y al mismo estímulo en general (Freud, 1979). Una de las propuestas se relaciona con que la satisfacción pulsional a través del consumo de sustancias podría deberse a la ausencia de un objeto a través del cual alcanzar la satisfacción, y por esta razón el objeto vuelve hacia la propia persona logrando la satisfacción placentera de forma pasiva a través del sujeto mismo; pudiendo generar consumos tanto experimentales y regulados, como problemáticos (Chaves, 2007).

El consumo de sustancias psicoactivas al funcionar como una distracción poderosa y una satisfacción sustitutiva, promueve la sublimación del sentimiento oceánico tan presente en las primeras etapas de desarrollo de la persona, volviendo al acto desde el yo en la estructura del aparato psíquico, y centrando todo en la vivencia propia de la satisfacción y dejando espacio para el trastorno hacia lo encontrando objeto y satisfacción pulsional en el mismo sujeto (Freud, 1930).

\section{Método}

El ejercicio investigativo se realizará con diseño cualitativo de investigación en el cual se emplea la observación con el fin de reconstruir la realidad, y desarrollar una descripción cercana a la realidad que se investiga (Belalcazar, Gonzalez-Arratia, Gurrola, y Moysén, 2002). Dentro de las técnicas a utilizar se realizarán dos etnografías en espacios de consumo recreativo, y entrevistas en profundidad con guion de la entrevista (Patton, 2002), la elección de participantes se realiza siguiendo un muestreo por intensidad de 5 personas que cumplan con los criterios de inclusión de tener entre 18 y 35 años cumplidos, ser consumidores de sustancias psicoactivas legales e ilegales, y presentar un consumo recreativo o regular. El análisis de los datos se realizará con una codificación axial (Hernández, 2014) tomando como ejes de análisis las cuatro dimensiones de la pulsión propuestas por Freud (1979).

\section{Resultados}

Como resultados preliminares se evidencia que la sustancia siempre funciona como objeto único para conseguir la meta deseada. Respecto a la fuente suele variar dependiendo del contexto o espacio recreativo en el cual se encuentre la persona, definiendo el espacio la sustancia más comúnmente utilizada, y llevando al consumo de esta misma. El esfuerzo se evidencia también como una característica asociada al espacio y suele incluir comportamientos y pensamientos orientados al uso del objeto y la consecución de la meta. 


\section{Discusión y Conclusión}

En términos teóricos de manera inicial se puede plantear que la pulsión es la fuerza que lleva a la búsqueda de la satisfacción, sin embargo, el objeto y el estimulo pulsional puede ser variables dependiendo siempre de la fuente. LA meta al mantenerse como un objetivo invariable no define si la sustancia, en este caso el objeto, es o no la correcta para alcanzar la satisfacción. El comportamiento de consumo cuando se vuelve compulsivo requiere de manera específica un acompañamiento terapéutico ya que se transforma en el trastorno hacia la propia persona excluyendo la fuente psíquica de la satisfacción en la meta de sólo uno de los componentes de la fuente.

\section{Referencias bibliográficas}

Belalcazar, P., Gonzalez-Arratia, N., Gurrola, G., y Moysén, A. (2002) Investigación cualitativa. México. Universidad Autónoma del Estado de México.

Chaves, D. (2007). Droga, psicoanálisis y toxicomanía. Las huellas de un encuentro. Desde el jardín de Freud(7), 317-318. Recuperado de: https://dialnet.unirioja.es/descarga/articulo/2923545.pdf

Daza-Orozco, C. E. (2019). Historia de la infancia en el cine colombiano (1st ed.). Retrieved from http://alejandria.poligran.edu.co/handle/10823/1648

Flórez-Romero Diana Paola; Castillo-Triana, Nicolás; Contreras-Ruiz, Paula Katerine; Henao-Floréz, Andrés; Ramírez-Toro, Diana Milena; Zárate-Pinto, Germán Camilo; Guzmán-Rodríguez, Rosa Julia; Anzelín-Zuluaga, Ingrid Carolina; Ruiz-He, Nicolás, R. G.-M. (2020). Fundamentos para la cualificación del talento humano: primera infancia y conflicto en Colombia (1st ed.). Bogotá: Institución Universitaria Politécnico Grancolombiano.

Freud, S. (1930). EL malestar en la cultura. Biblioteca libre Omegalfa. Recuperado de: https://www.omegalfa.es/autores.php?letra=f\#

Freud, S. (1979). Sigmund Freud obras completas volumen 14. Buenos Aires, Argentina: Amorrotu editores S. A.

Garrido, A. P. (2016). El Gran Juego de Asia Central se reencarna a comienzos del siglo XXI. Panorama, 10(18), 9-25. https://doi.org/10.15765/pnrm.v10i18.812

Guzmán, J. P. H. (2015). El Estado en la sociedad, explicación de cómo la disposición de las burocracias determina los órdenes en países periféricos. Panorama, 9(17), 104-122. https://doi.org/10.15765/pnrm.v9i17.795

Hernández, R. (2014). Metodología de la investigación. Mcgraw-Hill.

Obando, C. R. C. (2015). Movimientos barriales: reivindicación del derecho a la ciudad. Panorama, 9(16), 66-76. https://doi.org/10.15765/pnrm.vgi16.640

Observatorio de Drogas de Colombia. (2017). Reporte de drogas de Colombia. Ministerio de Justcia y del Derecho - Observatorio de Drogas de Colombia (ODC). Recuperado de: http://www.odc.gov.co/Portals/1/publicaciones/pdf/odc-libroblanco/reporte_drogas_colombia_2017.pdf

Oficina de las Naciones Unidas contra la Droga y el Delito. (2018). Informe mundial sobre las drogas 2018, 1. United Nations Publications. Recuperado de: https://www.unodc.org/wdr2018/prelaunch/WDR18 Booklet_1_EXSUM.pdf

Patton, M. (2002). Qualitative Research \& Evaluation Methods 3rd Edition. Thousand Oaks, CA: Sage Publications, Inc.

Peña, B. I. M., \& Herrera, L. K. D. (2016). Experiencia de cooperación en el acompañamiento empresarial integral en el territorio como aporte al proceso de reintegración en el posconflicto. Panorama, 10(18), 67-82. https://doi.org/10.15765/pnrm.v10i18.824 